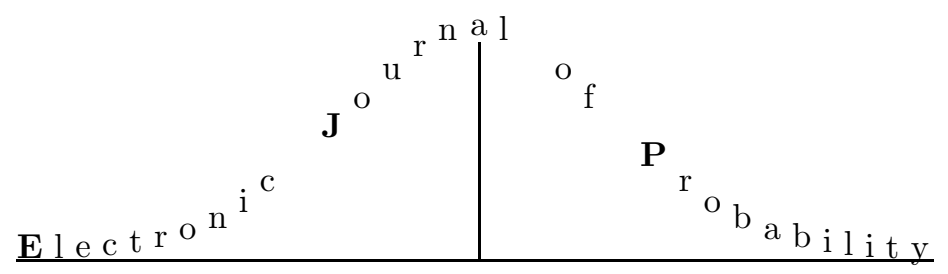

Vol. 12 (2007), Paper no. 37, pages 1048-1051.

Journal URL

http://www.math. washington.edu/ ejpecp/

\title{
Erratum to Eigenvalues of GUE Minors
}

\author{
Kurt Johansson $\quad$ Eric Nordenstam
}

\begin{abstract}
This is an erratum to EJP paper number 50, volume 11, Eigenvalues of GUE minors.
\end{abstract}

Key words: Random matrices; Tiling problems.

AMS 2000 Subject Classification: Primary 60G55; 15A52; 52C20.

Submitted to EJP on May 4, 2007, final version accepted July 20, 2007. 


\section{Introduction}

In the paper (JN06) two expressions for the so called GUE minor kernel are presented, one in definition 1.2 and one in the the formulas (5.6) and (5.7). The expressions given in (5.6) and (5.7) are correct, but the expression in definition 1.2 of the paper has to be modified in the case $r>s$. The proof of the equality of the two expressions for the GUE minor kernel given in the paper was based on lemma 5.6 which is not correct since some terms in the expansion are missing. The correct expansion is given in lemma 1.2 below.

The corrected version of definition 1.2 in $(\underline{\mathrm{JN06}})$ reads as follows.

Definition 1.1. The GUE minor kernel is

$$
K^{\mathrm{GUE}}(r, \xi ; s, \eta)=-\phi(r, \xi ; s, \eta)+\sum_{j=-\infty}^{-1} \sqrt{\frac{(s+j) !}{(r+j) !}} h_{r+j}(\xi) h_{s+j}(\eta) e^{-\left(\xi^{2}+\eta^{2}\right) / 2}
$$

where $\phi(r, \xi ; s, \eta)=0$ when $r \leq s$ and

$$
\begin{aligned}
\phi(r, \xi ; s, \eta)=\frac{(\xi-\eta)^{r-s-1} \sqrt{2^{r-s}}}{(r-s-1) !} e^{\frac{1}{2}\left(\eta^{2}-\xi^{2}\right)} H(\xi-\eta) \\
\quad-\frac{\frac{1}{2} e^{\left(\eta^{2}-\xi^{2}\right) / 2}}{\sqrt[4]{\pi}} \sum_{j=-r}^{-(s+1)} \frac{h_{r+j}(\xi) \sqrt{2^{-s-j}}}{\sqrt{(r+j) !}(-s-j-1) !} \int_{\eta}^{\infty}(t-\eta)^{-s-j-1} e^{-t^{2}} d t
\end{aligned}
$$

for $r>s$.

Here, $\left\{h_{k}\right\}_{k}$ are the normalised Hermite polynomials, defined to be $\equiv 0$ when $k<0$, and $H$ is the Heaviside function.

The expansion of the double integral formula (6.16) in (JN06) in terms of Hermite polynomials also has to be modified in the case $r>s$. This expansion was used in the proofs of lemma 3.15 and lemma 4.2 in (JN06) and the modification leads to the correct kernel given in definition 1.1 above. Expanding the integral formula in (6.16) gives

$$
\frac{\sqrt{2^{s-r} e^{\eta^{2}-\xi^{2}}}}{2(\pi i)^{2}} \sum_{k=0}^{\infty} \int_{\gamma} d u \frac{e^{2 \xi u-u^{2}}}{u^{r-k}} \int_{\Gamma} d v v^{s-k-1} e^{v^{2}-2 \eta v}
$$

where $\gamma$ is a small circle counter clockwise around the origin and $\Gamma$ is parameterised $v=2+i t$, where $t:-\infty \rightarrow \infty$. Here we recognize the two classical integral representations for the Hermite polynomials.

The proof in the article omitted the terms from $k=s+2$ to $k=r+1$ when $r>s$. Using the identity

$$
\frac{1}{\pi i} \int_{\Gamma} \frac{e^{v^{2}-2 \eta v}}{v^{n}} d v=\frac{2^{n}}{\sqrt{\pi}(n-1) !} \int_{\eta}^{\infty}(\xi-\eta)^{n-1} e^{-\xi^{2}} d \xi
$$

which is valid for $n \geq 1$ and one of the classical integral representation for the Hermite polynomials we obtain the expression we want for the omitted terms. 
It remains to verify the formula (2). Observe that, by repeated partial integration,

$$
\int_{\eta}^{\infty}(\xi-\eta)^{n-1} e^{-2 \xi v} d \xi=\frac{(n-1) ! e^{-2 \eta v}}{(2 v)^{n}}
$$

if $\eta>0$ and $v \in \Gamma$. So in this case the left hand side of our formula can be written

$$
\frac{2^{n}}{\pi i(n-1) !} \int_{\gamma} \int_{\eta}^{\infty}(\xi-\eta)^{n-1} e^{v^{2}-2 \xi v} d \xi d v .
$$

Here we can change the order of integration and evaluating the Gaussian integral gives the right hand side of (2).

When $\eta<0$, make a change of variables $v \mapsto-v$. The left hand side of (2) becomes

$$
\frac{(-1)^{n}}{\pi i} \int_{\Gamma^{\prime}} \frac{e^{v^{2}+2 \eta v}}{v^{n}} d v
$$

where $\Gamma^{\prime}$ is parameterised $v=-2+i t, t=-\infty \mapsto \infty$. By deforming the contour $\Gamma^{\prime}$ into $\Gamma$ we get

$$
\frac{(-1)^{n-1}}{\pi i} \int_{\gamma} \frac{e^{v^{2}+2 \eta v}}{v^{n}} d v+\frac{(-1)^{n}}{\pi i} \int_{\Gamma} \frac{e^{v^{2}-2(-\eta) v}}{v^{n}} d v
$$

where $\gamma$ is a circle around the origin. The right term can be evaluated using the the result for $\eta>0$ above. The left term can be rewritten using the equality between the two integral formulas for the Hermite polynomials mentioned above and we obtain

$$
\frac{2^{n}}{\sqrt{\pi}(n-1) !} \int_{-\infty}^{\infty}(\xi-\eta)^{n-1} e^{-\xi^{2}} d \xi-\frac{2^{n}}{\sqrt{\pi}(n-1) !} \int_{-\infty}^{\eta}(\xi-\eta)^{n-1} e^{-\xi^{2}} d \xi
$$

which proves formula (2) for $\eta<0$.

Finally, we give the corrected version of lemma 5.6 in (

Lemma 1.2. The following expansion

$$
\begin{aligned}
\frac{\sqrt{2^{k}}}{(k-1) !}(x-y)^{k-1} H(x-y)=\sum_{n=k}^{\infty} \sqrt{\frac{(n-k) !}{n !}} h_{n-k}(y) h_{n}(x) e^{-y^{2}} & \\
& +\frac{1}{\sqrt[4]{\pi}} \sum_{n=0}^{k-1} \frac{h_{n}(x) \sqrt{2^{k-n}}}{\sqrt{n !}(k-1-n) !} \int_{y}^{\infty}(t-y)^{k-1-n} e^{-t^{2}} d t
\end{aligned}
$$

holds pointwise for $x \neq y$.

Proof. Proceed as in the proof of lemma 5.6 in $(\mathrm{JN06})$ to compute $c_{n}(y)$ for $n \geq k$. That calculation fails for $0 \leq n<k$, and those terms account for the second sum in the statement of this lemma. Use the formula $e^{-x^{2}} H_{n}(x)=-\frac{d}{d x}\left(e^{-x^{2}} H_{n-1}(x)\right)$ to perform integration by parts as many times as possible.

$$
\begin{aligned}
\int_{y}^{\infty}(x-y)^{k-1} H_{n}(x) e^{-x^{2}} d x=\int_{y}^{\infty}(k-1) & (x-y)^{k-2} H_{n-1}(x) e^{-x^{2}} d x \\
& =\ldots=\frac{(k-1) !}{(k-n-1) !} \int_{y}^{\infty}(x-y)^{k-n-1} e^{-x^{2}} d x .
\end{aligned}
$$

Inserting this in (6.1) in ( lemma. 


\section{Acknowledgement}

We thank Peter Forrester and Taro Nagao for pointing out the mistake in the proof of lemma 5.6 in (JN06), indicating that terms were missing in the expression for the GUE minor kernel when $r>s$, and sending us a preliminary version of work in progress.

\section{References}

[JN06] Kurt Johansson and Eric Nordenstam. Eigenvalues of GUE minors. Electron. J. Probab., 11(50):1342-1371 (electronic), 2006. 